\title{
Transcriptional Regulation by the Spalt Proteins: Filling Up the Gaps
}

\section{Jose F de Celis* and Cristina M Ostalé}

Severo Ochoa Molecular Biology Center, Nicolás Cabrera, Madrid, Spain

Differential gene expression is the most versatile and employed mechanism to generate cell diversity during development [1]. There are many macromolecular complexes that impinge on transcription, including the general transcription machinery [2], chromatin modifying enzymes driving histone acetylation, methylation or phosphorylation [3], nucleosome remodelers [4] and sequence-specific Transcription Factors (TF; $[5,6]$. These last proteins provide specificity to the regulation of gene expression by binding to 6-10 bp DNA sequences present in the regulatory regions of their target genes, where they recruit other transcriptional activators or repressors [7]. There are many instances during development where TFs function in complex networks in which each TF is part of a combinatorial code regulating the expression of one or very few downstream genes present in a Gene Regulatory Networks, GRN) [8]. Perhaps the most paradigmatic example occurs during Drosophila embryonic segmentation, where a hierarchy of TFs generates progressively smaller and periodic domains of gene expression [9]. In segmentation, as in many other examples, TFs arranged in GRN contribute to generate ordered spatio-temporal transcriptional heterogeneities in a developing tissue [10]. The components, structure and regulatory logic of such networks can be dissected through the use of Chromatin Immunoprecipitation (ChIP) and genome-wide gene expression assays combined with genetic analyses and a variety of reporter expression experiments [9].

In contrast to TFs with high DNA sequence specificity binding and few target downstream genes, other TFs display lower binding specificity and affect the expression of large sets of target genes [11]. In these last cases, it is much more difficult to determine their molecular functions, which ultimately depends on the identification of the genes they regulate, the sequences they bind and the mechanism of transcriptional regulation in which they participate. Examples of these TFs are the Hox genes, a conserved set of proteins with homeodomain DNA binding motives that determine morphological differences among homologous structures [12]. These TFs were considered as master regulators because of the systemic effects of their mutations, but more recently they were described as "micromanagers", due to their ability to regulate the expression of a multiplicity of targets [13]. The Hox TFs show pervasive DNA binding and cause quantitatively weak effects on the expression levels of a large number of target genes [14]. Another family of TFs showing similar characteristics to the Hox genes are the $\underline{S}$ alt proteins (Sal). These proteins are conserved from worms to humans, and they contain several pairs of zinc finger domains and a Glutamine rich region [15]. Human Sal genes (Sall1-4) had attracted considerable attention because of their involvement in the genetic diseases Townes Brocks syndrome (SALL1) and Okihiro syndrome (Sall4), their implication in a variety of cancers (Sall2) and their developmental roles during stem cell formation and organogenesis $[15,16]$.

The sal genes were originally identified in flies, where they participate in a variety of developmental processes including trachea and limb formation and neural determination $[15,17]$. They play a key role in the formation of the fly wing, acting downstream of the BMP signalling pathway to regulate vein patterning and to modulate general cellular properties in broad cellular territories [18]. The function of Sal proteins in patterning suggests that they act as members of the GRNs regulating the subdivision of the wing epithelium in gene expression domains [11], in a process that bears strong similarities with embryonic segmentation [11]. Interestingly, the Sal transcription factors are also required for the modulation of cell division, adhesion, survival and polarity in the central region of the wing, their territory of expression [18]. A recent work indicates that the Spalt proteins regulate the expression of multitude of target genes in the wing epithelium $[19,20]$ (Figure 1). We assume that it is the ability of these proteins to interact with multiple cofactors and DNA sequences what confers a rich versatility to their biological functions.

Understanding how the Sal proteins combine a general impact on transcription during the formation of the central region of the wing with exquisite precision and target specificity during vein patterning requires knowing how they regulate gene expression. To date, several studies suggest that Sal proteins act as transcriptional repressors [21] and that they exert this activity by recruiting epigenetic modifiers. For example, the mouse ortholog Sall1 behaves as a direct negative regulation of transcription [19] and transcriptional repression by Sall1 in luciferase assays is reduced by the Histone deacetylase (HDAC) inhibitor Trichostatin A (TSA). This observation suggests that Sall1 transcriptional repression is HDAC-dependent. In fact, Sall1 interacts with HDAC1, HDAC2 and RbAp46/48 (common components of the NuRD and Sin $3 \mathrm{~A}$ deacetylase complexes), and also with MTA1/2 (specific component of NuRD complexes). Subsequently, it was show that Sall1 and Sall4 interact with Mi2/NuRD chromatin remodelling complexes [22,23]. In addition to deacetylases, Sall4 is also able to recruit the lysine-specific histone demethylase 1 to its transcriptional targets in adult mouse bone marrow cells where it has a critical role in haematopoiesis [23]. All this results emphasize the importance of histone-tail modifications in the transcriptional repression mediated by Sal proteins. However, it is still unclear what DNA sequences are recognised by Sal transcription factors to bring these complexes to specific genomic regions. Although it has not yet been possible to define a unique consensus DNA binding sequence for Sal proteins, it is known that an A/T-rich region is necessary for Sall1 binding in mouse embryonic stem cells and fibroblast [24] and an A/T-rich sequence is also required for Drosophila Spalt-related DNA binding in vitro [17]. Also, Sall2 can activate the expression of p16 by binding to a $\mathrm{G} / \mathrm{C}$-rich region in its promoter [25], but the precise sequences and in vivo mechanism by which Sal proteins recognize and bind to its cis-regulatory elements reminds to be clarified. It is most likely that Sal cofactors play a key role in determining target specificity. An additional complication, which is not in full agreement with Sal function as sequence-specific TFs, is that these proteins have been found to localise in pericentromeric heterochromatic regions of DNA [21] Furthermore, SALL1 partially localises with M31, the mouse homolog of the Drosophila heterochromatic protein HP1, and interacts with an isoform of the telomere-repeat-binding factor 1 (TRF1) $[21,24]$. These

*Corresponding author: Jose F de Celis, Higher Council for Scientific Research and Autonomous University of Madrid, Severo Ochoa Molecular Biology Center, Nicolás Cabrera, Madrid, Spain, E-mail: jfdecelis@cbm.csic.es

Received September 13, 2017; Accepted September 19, 2017; Published September 26, 2017

Citation: de Celis JF, Ostalé CM (2017) Transcriptional Regulation by the Spalt Proteins: Filling Up the Gaps. Mol Biol 6: 196. doi: 10.4172/2168-9547.1000196

Copyright: () 2017 de Celis JF, et al. This is an open-access article distributed under the terms of the Creative Commons Attribution License, which permits unrestricted use, distribution, and reproduction in any medium, provided the original author and source are credited. 


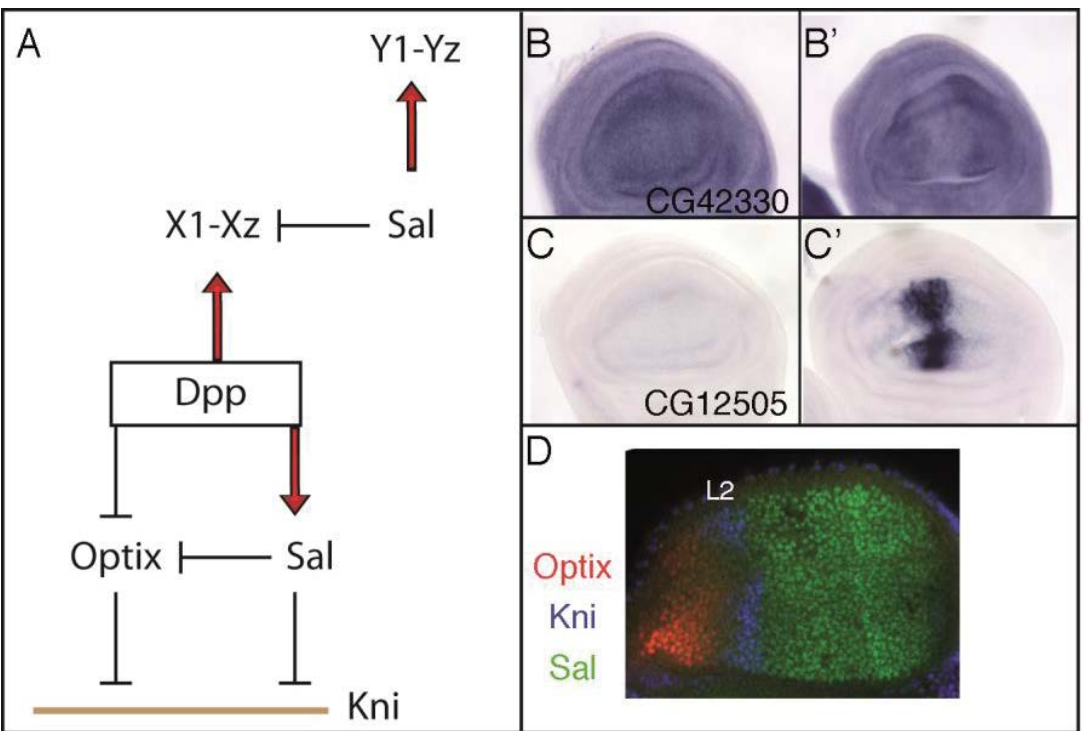

Figure 1: Regulatory networks in which the Spalt proteins participate during the development of the Drosophila wing.

(A) Regulatory networks involved in the positioning of the L2 vein (Dpp; decapentaplegic (Dpp) signalling, Optix, Sal and Kni), and other gene regulatory processes requiring Spalt and involving the activation or repression of a variety of Sal target genes ( $Y 1$ to $Y z$ and $X 1$ to $X z$, respectively). (B-B') Example of a gene (CG42330) requiring Sal activity for normal expression in the central region of the wing blade epithelium. The normal expression of the gene (B) is reduced in the centre of the wing blade in sal mutant conditions (B'). (C-C') Example of a gene (CG12505) repressed by Sal activity in the central region of the wing blade epithelium. The gene is normally not expressed in the wing disc $(C)$ but expressed at high levels in the central region of the wing blade in sal mutant condition ( $\left.C^{\prime}\right)$. Images in $B-C^{\prime}$ correspond to in situ hybridization with probes for the genes CG42330 (B-B') and CG12505 (C-C'), in wild type discs (B and C) and in sal mutant discs (B'and C'). (D) Expression of Sal (green), Optix (red) and Kni (Blue in the wing blade region of the wing disc.

observations suggest that Sal proteins might play a more generalized role in the generation or maintenance of heterochromatin, and affect gene expression by acting as determinants of chromatin structure. The identification of a large set of Sal downstream genes opens the possibility of identifying the DNA sequences mediating this regulation, as well as the cofactors and mechanisms by which Sal regulates gene expression [26,27].

\section{Acknowledgement}

We thank Carlos Estella for critical reading of the manuscript. This work was supported by MINECO grant BFU2015-64220-P to JFdC. Institutional support from Fundación Ramón Areces and Banco de Santander is also acknowledged.

\section{References}

1. Arnone MI, Davidson EH (1997) The hardwiring of development: organization and function of genomic regulatory systems. Development 124: 1851-1864.

2. Sainsbury S, Bernecky C, Cramer $P$ (2015) Structural basis of transcription initiation by RNA polymerase II. Nat Rev Mol Cell Biol 16: 129-143.

3. Allis C, Jenuwein T (2016) The molecular hallmarks of epigenetic control. Nat Rev Genet 17: 487-500.

4. Clapier C, Cairns B (2009) The biology of chromatin remodeling complexes Annu Rev Biochem 78: 273-304.

5. Koster M, Snel B, Timmers H (2015) Genesis of chromatin and transcription dynamics in the origin of species. Cell 161: 724-736.

6. Levine M, Cattoglio C, Tjian R (2014) Looping back to leap forward: Transcription enters a new era. Cell 161: 724-736.

7. Slattery M, Zhou T, Yang L, Machado A, Gordan R, et al. (2014) Absence of a simple code: How transcription factors read the genome. Trends Biochem Sci 39: 381-399

8. Levine M, Davidson E (2005) Gene regulatory networks for development PNAS 14: 4936-4942.

9. Levine M (2008) A systems view of Drosophila segmentation Genome Biol 9: 207.
10. Martín M, Organista M, JF d (2016) Structure of developmental gene regulatory networks from the perspective of cell fate-determining genes. Transcription 7 : 32-37

11. Martin M, Organista M F, de Celis (2016) Structure of developmental gene regulatory networks from the perspective of cell fate-determining genes. Transcription 7: 32-37.

12. Mallo M, Wellikcd D, Deschampse J (2010) Hox genes and regional patterning of the vertebrate body plan. Developmental Biology 344: 7-15.

13. Akam M (1998) Hox genes: From master genes to micromanagers. Curr Biol 8: $676-678$.

14. Rezsohazy R, Saurin A, Maurel-Zaffran C, Graba Y (2015) Cellular and molecular insights into Hox protein action. Development 142: 1212-1227.

15. de Celis JF, Barrio R (2009) Regulation and function of Spalt proteins during animal development. Int J Dev Biol 53: 1385-1398.

16. Sweetman D, Münsterberg A (2006) The vertebrate spalt genes in development and disease. Dev Biol 293: 285-293.

17. Barrio R, Shea MJ, Carulli J, Lipkow K, Gaul U, et al. (1996) The spalt-related gene of Drosophila melanogaster is a member of an ancient family defined by the adjacent, region-specific homeotic gene spalt. Dev Genes Evol 206: 315-325.

18. Organista M F and De Celis J F (2013) The Spalt transcription factors regulate cell proliferation, survival and epithelial integrity downstream of the Decapentaplegic signalling pathway. Biol Open 2: 37-48.

19. Kiefer S M, McDill B W, Yang J, Rauchman M (2002) Murine Sall1 represses transcription by recruiting a histone deacetylase complex. J Biol Chem 277 : 14869-14876.

20. Sánchez J, Talamillo A, González M, Sánchez-Pulido L, Jiménez S, et al (2011) Drosophila Sal and Salr are transcriptional repressors. Biochem J 438 437-445.

21. Netzer C, Rieger L, Brero A, Zhang C, Hinzke M, et al (2001) SALL1, the gene mutated in Townes-Brocks syndrome, encodes a transcriptional repressor which interacts with TRF1/PIN2 and localizes to pericentromeric heterochromatin. Hum Mol Genet 10: 3017-3024. 
Citation: de Celis JF, Ostalé CM (2017) Transcriptional Regulation by the Spalt Proteins: Filling Up the Gaps. Mol Biol 6: 196. doi: 10.4172/21689547.1000196

22. Lauberth SM, Rauchman M (2006) A conserved 12-amino acid motif in Sall1 recruits the nucleosome remodeling and deacetylase corepressor complex. Biol Chem 281: 23922-31.

23. Lu J, Jeong HW, Kong N, Yang Y, Carroll J, et al. (2009) Stem cell factor SALL4 represses the transcriptions of PTEN and SALL1 through an epigenetic repressor complex. PloS ONE 4: 5577.

24. Yamashita K, Sato A, Asashima M, PC W, Nishinakamura R (2007) Mouse homolog of SALL1 a causative gene for Townes-Brocks syndrome binds to A/T-rich sequences in pericentric heterochromatin via its Cterminal zinc finger domains. Genes Cells 12: 171-182.
25. Wu Z, Cheng K, Shi L, Li Z, Negi H, et al. (2015) Sal-like protein 2 upregulates p16 expression through a proximal promoter element. Cancer Sci 106: 253261

26. Martín M, Ostalé C, de Celis J (2017) Patterning of the Drosophila L2 vein is driven by regulatory interactions between region-specific transcription factors expressed in response to Dpp signalling. Development 144: 3168-3176.

27. Organista M, Martín M, JM d C, Barrio R, López-Varea A, et al. (2015) The Spalt Transcription factors generate the transcriptional landscape of the Drosophila melanogaster wing pouch central region. PLoS Genet 11: 1005370. 